\title{
CONSUMO DE SUSTANCIAS ADICTIVAS Y EFECTOS EN LAS RELACIONES INTERGRUPALES $Y$ PERSONALES EN ESTUDIANTES UNIVERSITARIOS
}

\author{
María Esther Gallardo Pastrana, Carmen María Manrubia Prados, Raquel Muñoz \\ Palomino. Universidad de Granada
}

Fecha de recepción: 28 de mayo de 2012

Fecha de aceptación: 11 de junio de 2012

Localizador: http:www.ugr.es/local/miguelgr/ReiDoCrea-Vol.1-Art.11-Gallardo-Manrubia-Munoz.pdf

\section{RESUMEN}

Tras una primera revisión bibliográfica se llega a la conclusión de que, en el ámbito del estudiante universitario español, no se han realizado estudios acerca de las consecuencias del consumo de sustancias adictivas en relación a las relaciones interpersonales. Nuestro objetivo es analizar los efectos del consumo de sustancias adictivas dentro de las relaciones intergrupales en el ámbito universitario para que, posteriormente, esta investigación pueda ser llevada a cabo en otros ámbitos. La muestra empleada, que ha sido seleccionada de manera aleatoria, queda organizada en 50 estudiantes, a los cuales se les ha pasado un cuestionario relacionado con el tema investigado. Nuestros resultados apoyan el efecto que causa el consumo de sustancias adictivas, a través de las diferentes variables estudiadas, en la población universitaria.

PALABRAS CLAVES: Sustancias adictivas, consumo, consecuencia, relaciones intergrupales género, concienciación.

\begin{abstract}
After an initial literature review it was concluded that, in the sphere of Spanish university students, there have been no studies on the consequences of addictive substance use in relation to interpersonal relationships. Our goal is to analyze the effects of substance use on intergroup relationships in the university so that this research can be carried out subsequently in other areas. The sample, which was selected at random, is composed of 50 students, who completed a questionnaire on the subject investigated. Our results confirm the effect of substance abuse, through the different variables studied, in the university population.
\end{abstract}

KEYWORDS: Addictive substances, consumption, consequences, intergroup relations, gender,awareness. 


\section{INTRODUCCIÓN}

El consumo de sustancias adictivas, tanto legales como ilegales, está estudiado en datos epidemiológicos sistemáticos por el Plan Nacional sobre Drogas, lo que ha generado la presencia de estudios rigurosos al respecto. Según la Encuesta Domiciliaria sobre Alcohol y Drogas en España (EDADES) 2009/2010 ejecutada por la Delegación del Gobierno para el Plan Nacional sobre Drogas muestra que las drogas más consumidas son el alcohol (84,4\% hombres y $72,7 \%$ mujeres), el tabaco $(48,4 \%$ hombres y $37,0 \%$ mujeres), el cannabis (14,8\% hombres y $6,2 \%$ mujeres) y los hipnosedantes (4,6\% hombres y $9,4 \%$ mujeres). En donde tenemos que el alcohol es la sustancia psicoactiva más consumida (79\%) y el cannabis es la droga ilegal más requerida (11\%).

En España estos estudios no han tenido en cuenta de forma específica la población universitaria, ya que el Plan Nacional sobre Drogas lo ha hecho de forma generalizada en una población comprendida entre los 15-64 años. Por este motivo nuestro estudio se centra en estudiantes universitarios y su consumo de drogas, tanto legales como ilegales y en la diferenciación entre sexos (hombres y mujeres) del consumo.

A estos estudios epidemiológicos añadiremos la variable relacional entre las relaciones intergrupales y personales y una mayor profundización en las consecuencias del consumo de sustancias adictivas (legales e ilegales). Variables que tienen una gran relevancia en el plano estudiantil en nivel universitario.

Las diferentes variables que hemos tenido que operativizar para estudiarlas son: sustancias adictivas, estudiantes universitarios, relaciones intergrupales, relaciones personales, consecuencias del consumo de sustancias adictivas (legales e ilegales) y consumo de drogas en los dos géneros (masculino y femenino). Las hemos operativizado mediante un cuestionario cerrado.

Los objetivos de este estudio son conocer el tipo de sustancias adictivas que más consumen los estudiantes universitarios, reflejar los efectos en las relaciones intergrupales y personales, profundizar en las consecuencias del consumo de sustancias adictivas y diferenciar el consumo de drogas, tanto legales como ilegales, entre hombres y mujeres.

Los resultados que se esperan obtener son que, cuanto más nivel de consumo de sustancias adictivas haya entre el sector universitario, mayores serán las relaciones negativas intergrupales y personales. Igualmente, se espera que la no concienciación del consumo de sustancias adictivas sea el efecto de un mayor consumo. Por último, se cree que el consumo de sustancias adictivas es mayor entre el género masculino que el género femenino.

\section{MÉTODO}

\section{Sujetos}

La muestra está constituida por un total de 50 sujetos seleccionados de manera aleatoria, con edades comprendidas entre los 21 y 53 años. Los sujetos pertenecen a la Facultad de Ciencias de la Educación de la Universidad de Granada. 


\section{Instrumentos}

Las variables planteadas han sido evaluadas mediante un cuestionario formado por una pregunta de opción múltiple en la que los sujetos podían contestar varias elecciones de las doce planteadas, una segunda pregunta de respuesta abierta y finalmente, veintitrés preguntas de respuesta dicotómica en las que los sujetos debían indicar si estaban de acuerdo o no con la cuestión planteada.

En dicho cuestionario, la variable tipo de sustancias adictivas se va a evaluar en la pregunta $A$ y en el apartado B se evaluará la frecuencia de consumo. El consumo de sustancias adictivas se evalúa en los ítems 8-13, 14 y 17-20; las consecuencias del consumo se aprecian en los ítems 3-6, 15, 16 y 21; y, las relaciones interpersonales se evalúan a partir de los ítems 1, 2 y 17-20. Decir que todos los ítems se van a evaluar en relación al género.

El instrumento utilizado lo hemos adaptado de acuerdo a nuestros intereses en la presente investigación. Está en vías de validación y ha sido extraído de la página web http://es.scribd.com.

\section{Procedimiento}

Los distintos cuestionarios fueron cumplimentados en la semana del 14 al 19 de mayo del 2012. A cada sujeto se le entregó un documento con el cuestionario, siendo éste rellenado de manera individual de acuerdo a las instrucciones que en él se recogen.

\section{RESULTADOS}

El tipo de sustancias adictivas que consumen los sujetos queda recogido en la siguiente gráfica:

\section{Tipo de sustancia}

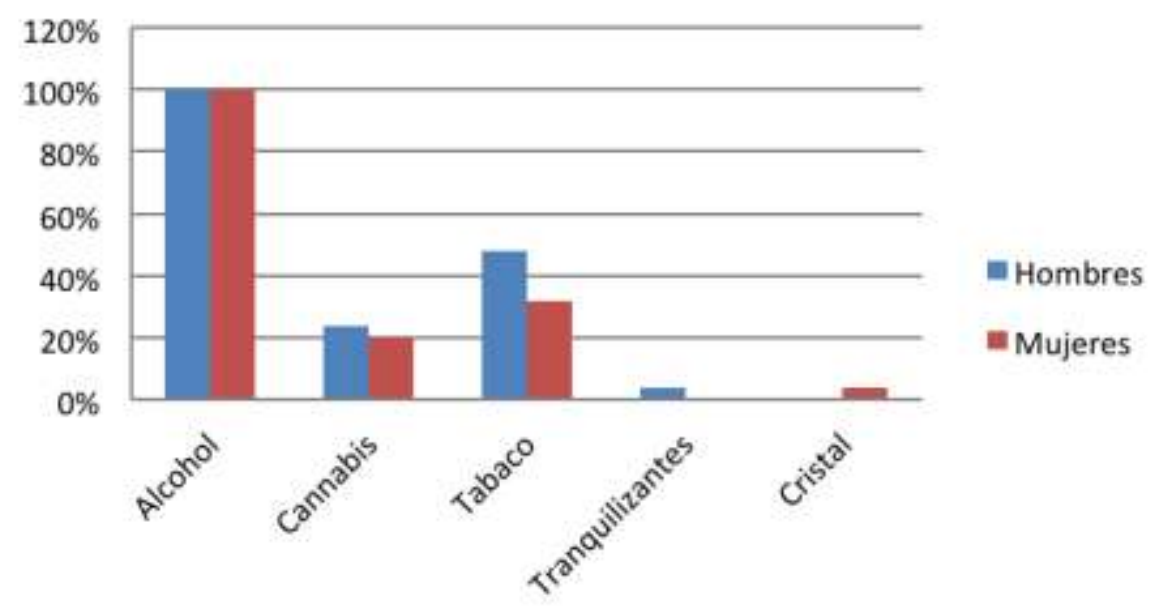

En dicha gráfica se han recogido los datos de aquellas sustancias que los sujetos han consumido en alguna ocasión. Como se puede apreciar, el total de los sujetos han afirmado haber consumido alcohol en alguna ocasión, seguido del consumo del tabaco, cuyos datos ascienden a un $48 \%$ para los hombres y un $32 \%$ para las mujeres. 
En cuanto al consumo del cannabis, los datos reflejan que el $24 \%$ de los hombres y el $20 \%$ de las mujeres, han consumido en alguna ocasión dicha sustancia, ocupando el tercer lugar de acuerdo a la sustancia más consumida.

En relación a tranquilizantes y cristal, solo uno de los sujetos en cada caso ha marcado dicha opción, siendo un hombre en el primer caso y una mujer en el segundo.

La siguiente gráfica hace alusión al consumo de sustancias relacionadas, como en el anterior caso, con la variable género:

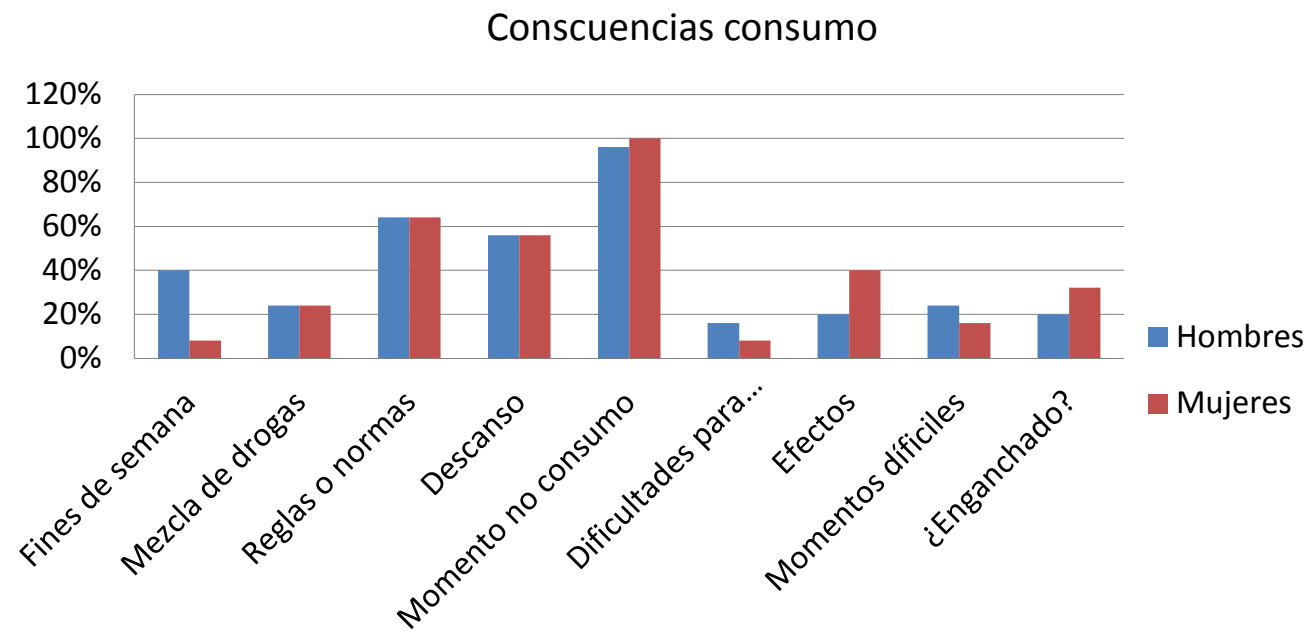

Los resultados de esta gráfica resaltan que, tanto hombres como mujeres, establecen reglas o normas tanto para el consumo como para el descanso de éste, siendo algo mayor en el género femenino el momento de no consumo.

En la variable dificultades para decir no, se puede apreciar que la mayoría de sujetos encuestados no presentan dificultades a la hora de tener que decir no al consumo de sustancias adictivas.

La tercera gráfica que recogemos hace alusión a las consecuencias que se derivan del consumo de sustancias relacionándolas también con la variable género:

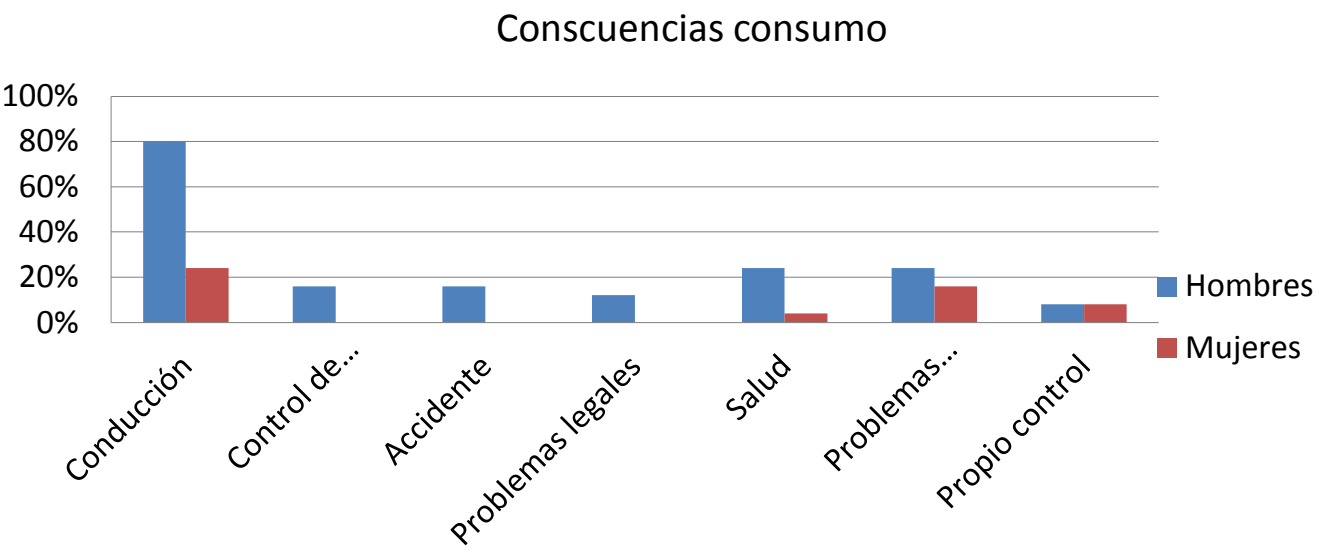


En esta gráfica se aprecia, en la mayoría de variables, mayores consecuencias en el género masculino, no apreciándose éstas en el caso de control de alcoholemia, accidentes de tráfico y problemas legales en el género femenino.

Destacar el hecho de que el $80 \%$ de los sujetos masculinos encuestados han conducido bajo los efectos de alguna sustancia, siendo el porcentaje de mujeres del $24 \%$.

En cuanto a problemas psicológicos, de salud o de propio control, se aprecia que en ninguno de los casos sobrepasa el $25 \%$ de los sujetos encuestados, siendo en los dos primeros casos la cifra mayor en los hombres, y en el último caso igual para ambos géneros.

Por último, incluir una gráfica con respecto a la variable relaciones interpersonales, estudiada en comparación con la variable género:

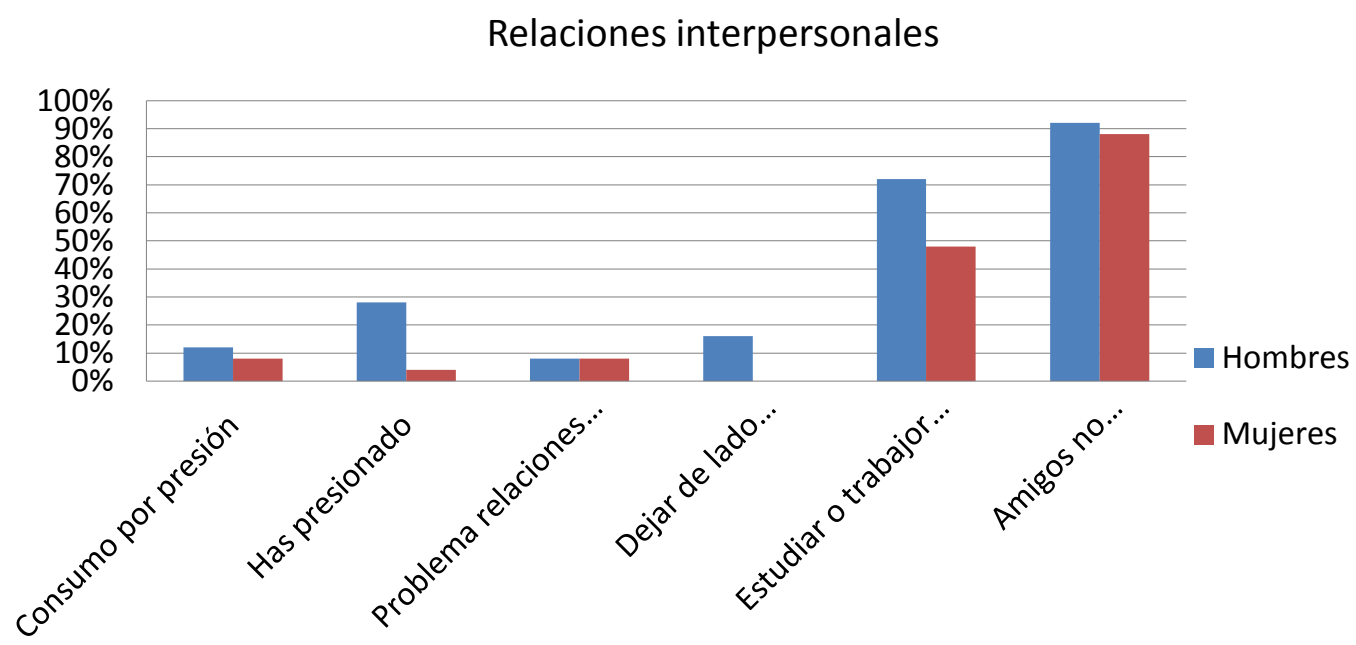

En esta última gráfica destaca el porcentaje de la variable amigos no consumidores, quedando reflejado que la mayoría de los sujetos encuestados se relacionan con personas de esta índole.

Es de destacar el alto porcentaje obtenido en la variable estudiar o trabajar bajo los efectos de alguna sustancia en la que más del $60 \%$ del total de sujetos encuestados, ha contestado positivamente a la misma.

En la variable dejar de lado actividades se observa que un $16 \%$ de los sujetos encuestados de género masculino, ha contestado afirmativamente a esta cuestión y, en cambio, en el género femenino no se ha obtenido ninguna contestación afirmativa.

\section{DISCUSIÓN}

Concluimos que las tres hipótesis planteadas, cuanto más nivel de consumo de sustancias adictivas haya entre el sector universitario, mayores serán las relaciones negativas intergrupales y personales; la no concienciación del consumo de sustancias adictivas sea el efecto de un mayor consumo; y, el consumo de sustancias adictivas es mayor entre el género masculino que en el género femenino, cumplen nuestras 
expectativas ya que los resultados del análisis estadístico realizado a las encuestas, apoyan las mismas.

Por dar respuesta a la no existencia de investigaciones acerca del consumo de sustancias adictivas en correspondencia con las relaciones intergrupales en el ámbito universitario español, hemos encontrado el inconveniente de que no se puede extrapolar los resultados obtenidos a otra población ya que la muestra seleccionada aleatoriamente se ha centrado en un contexto específico y tiene un tamaño reducido.

Además de analizar, obtener y publicar la presente investigación, se va a hacer llegar a todos los sujetos seleccionados, los resultados que se han obtenido en la misma.

Para concluir, esta investigación servirá de base a futuras exploraciones, pudiendo ser utilizada en diferentes ámbitos y con una población diversa.

\section{BIBLIOGRAFÍA}

Encuesta Domiciliaria sobre Alcohol y Drogas en España (EDADES) 2009/2010. http://www.ine.es/ioe/ioeFicha.jsp?cod=54102, consultada el día 18 de mayo de 2012.

Encuesta acerca del consumo de sustancias adictivas, consultada en http://es.scribd.com/search?query=encuesta, el día 8 de mayo de 2012.

Conrado, M. Viña y Herrero, Manuel (2004). El consumo de sustancias psicoactivas en estudiantes de psicología de la Universidad de la Laguna. International Journal of Clinical and Health Psychology, 4(3), 521-536.

Jiménez-Muro Franco, A., Beamonte San Agustín, A., Marqueta Baile, A., Gargallo Valero, P. y Nerín de la Puerta, I. (2009). Consumo de drogas en estudiantes universitarios de primer curso. Adicciones (1), 21-28. 


\section{ANEXO}

\section{Instrumento de recogida de datos}

A continuación te presentamos un cuestionario y te pedimos que seas lo mas sincero/a posible en tus contestaciones ya que estás formando parte de una investigación. Antes de pasar a contestar, no olvides anotar tu sexo y edad. ¡Gracias por tu participación!

Sexo:

Edad:

¿Qué tipos de drogas consumes? (Puedes marcar más de una opción)

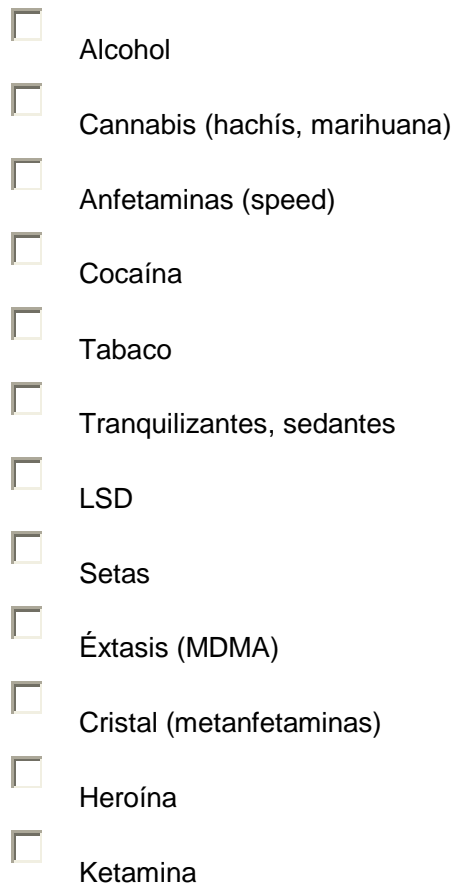


¿Cuál es la sustancia principal o que consumes más frecuentemente?

\begin{tabular}{|c|c|c|}
\hline Ítem & Sí & No \\
\hline ¿Has consumido alguna vez una droga por sentirte presionado? & & \\
\hline ¿Has presionado alguna vez a alguien para que consuma alguna droga? & & \\
\hline ¿Has conducido el coche o la moto bajo los efectos de alguna sustancia? & & \\
\hline ¿Alguna vez has dado positivo en un control de tráfico de alcoholemia-drogas? & & \\
\hline ¿Has tenido algún accidente relacionado con el consumo de drogas? & & \\
\hline ¿Has tenido alguna vez problemas legales por consumo o posesión de drogas? & & \\
\hline ¿Sueles utilizar preservativo en tus relaciones sexuales cuando estás de marcha? & & \\
\hline ¿Consumes todos los fines de semana? & & \\
\hline ¿Sueles mezclar diferentes drogas? & & \\
\hline $\begin{array}{l}\text { ¿Tienes reglas o normas sobre tu propio consumo? (Consumir solo en lugares concretos, momentos puntuales, } \\
\text { no tomar más que lo planeado, etc.). }\end{array}$ & & \\
\hline ¿Estableces momentos o temporadas de descanso en tu consumo? & & \\
\hline ¿Tienes claro cuando es el momento de no consumir? (Por ejemplo cuando al día siguiente tienes un examen). & & \\
\hline ¿Te cuesta trabajo o tienes dificultades para decir "no"? & & \\
\hline ¿Sueles consumir de forma que notas los primeros efectos hasta tomar otra dosis? & & \\
\hline $\begin{array}{l}\text { ¿Te has pasado alguna vez, hasta el punto que hayas tenido consecuencias negativas para tu salud? (Como } \\
\text { etílico, ingreso hospitalario, lipotimia, etc.). }\end{array}$ & & \\
\hline $\begin{array}{l}\text { ¿Has sufrido problemas psicológicos debidos al consumo de alguna sustancia? (Angustia, ansiedad, insomnio, } \\
\text { dependiente, etc.). }\end{array}$ & & \\
\hline ¿Has continuado consumiendo a pesar de presentar problemas para tus relaciones sociales? & & \\
\hline $\begin{array}{l}\text { ¿Has descuidado o suspendido actividades importantes como estudios, deportes, trabajo, compartir con amigos } \\
\text { o familiares, por consumir alguna sustancia? }\end{array}$ & & \\
\hline ¿Alguna vez has ido a trabajar o estudiar bajo los efectos de alguna sustancia? & & \\
\hline ¿Tienes amigos o grupo de amigos que no sean consumidores? & & \\
\hline ¿Alguna vez has perdido el control hasta el punto de no poder parar de consumir? & & \\
\hline $\begin{array}{l}\text { ¿Has consumido drogas en algún momento o época de tu vida en que lo estabas pasando mal, para afrontar } \\
\text { tus problemas o evitarlos? }\end{array}$ & & \\
\hline a ocasión que te estabas "enganchando" al consur & & \\
\hline
\end{tabular}

